\title{
Neural correlates of explicit and implicit emotion processing in relation to treatment response in pediatric anxiety
}

\author{
Katie L. Burkhouse, ${ }^{1}$ Autumn Kujawa, ${ }^{1}$ Heide Klumpp, ${ }^{1,2}$ Kate D. Fitzgerald, ${ }^{3}$ \\ Christopher S. Monk, ${ }^{3,4}$ and K. Luan Phan ${ }^{1,2,5}$ \\ ${ }^{1}$ Department of Psychiatry, University of Illinois at Chicago, Chicago, IL; ${ }^{2}$ Department of Psychology, University of \\ Illinois at Chicago, Chicago, IL; ${ }^{3}$ Department of Psychiatry, University of Michigan, Ann Arbor, MI; ${ }^{4}$ Department of \\ Psychology, University of Michigan, Ann Arbor, MI; ${ }^{5}$ Department of Anatomy and Cell Biology, and Graduate Program \\ in Neuroscience, University of Illinois at Chicago, Chicago, IL, USA
}

\begin{abstract}
Background: Approximately $40 \%-45 \%$ of youth with anxiety disorders do not achieve remission (or a substantial reduction in symptoms) following treatment, highlighting the need to identify predictors of treatment response. Given the well-established link between attentional biases and anxiety disorders in youth and adults, this study examined the neural correlates of directing attention toward and away from emotional faces in relation to pediatric anxiety treatment response. Method: Prior to beginning treatment with the selective serotonin reuptake inhibitor (SSRI) sertraline or cognitive behavior therapy (CBT), 37 youth (age 7-19 years) with generalized and/or social anxiety disorder completed a task with conditions that manipulated whether participants were instructed to match emotional faces (explicit emotion processing) or match shapes in the context of emotional face distractors (implicit emotion processing) during functional magnetic resonance imaging. Results: Results revealed that reduced activation in superior frontal gyrus (SFG), encompassing the dorsal anterior cingulate cortex (ACC) and dorsomedial prefrontal cortex $(\mathrm{PFC})$, during implicit processing of emotional faces predicted a greater reduction in anxiety severity pre-to-post treatment. Post hoc analyses indicated that effects were not significantly moderated by the type of treatment or anxiety type. Conclusions: Findings suggest that less recruitment of SFG, including the dorsal ACC and dorsomedial PFC, during implicit emotion processing predicts a greater reduction in youth anxiety symptoms pre-topost treatment. Youth who exhibit reduced activation in these areas while matching shapes in the context of emotional face distractors may have more to gain from CBT and SSRI treatment due to preexisting deficits in attentional control. These findings suggest that neuroimaging may be a useful tool for predicting which youth are most likely to benefit from anxiety treatment. Keywords: Pediatric anxiety; treatment response; neuroimaging; attention; emotion processing.
\end{abstract}

\section{Introduction}

Pediatric anxiety disorders, affecting approximately $10 \%-15 \%$ of children and adolescents (Costello, Mustillo, Erkanli, Keeler, \& Angold, 2003), are one of the most common classes of psychological disorders in youth and are associated with impairment in social and academic functioning, and increased risk of multiple disorders later in life (Mychailyszyn, Méndez, \& Kendall, 2010). Although pediatric anxiety can be treated effectively through the use of cognitive behavioral therapy (CBT) and pharmacological treatments [i.e. selective serotonin reuptake inhibitors (SSRIs)], approximately $40 \%-45 \%$ of youth do not achieve remission or a substantial reduction in symptoms (Mohatt, Bennett, \& Walkup, 2014). Identifying predictors of outcome may allow clinicians to identify youth at risk for poorer outcomes and help guide patients toward those treatments with the highest likelihood of success.

Cognitive models of anxiety suggest that individuals' characteristic ways of attending to threatening stimuli contribute to the development, maintenance,

Conflict of interest statement: No conflicts declared. and treatment of the disorder (e.g. Clark, Beck, \& Alford, 1999). Supporting these models, studies demonstrate that anxiety disorders in children and adults are characterized by both initial orienting of attention to threatening (angry or fearful) stimuli and difficultly diverting attention from threatening stimuli (e.g. Bar-Haim, Lamy, Pergamin, BakermansKranenburg, \& Van Ijzendoorn, 2007; Bishop, 2007; Pine, 2007). Studies investigating the neural correlates of these attentional responses in anxiety highlight overactivation of regions associated with the detection of threat, such as the amygdala, and altered top-down control by regions associated with goal-directed attention, such as the lateral prefrontal cortex $(\mathrm{PFC})$ and anterior cingulate cortex (ACC) (Bishop, 2007). Engagement of the ACC, in particular, appears to be critical in maintaining an appropriate balance between sensitivity to potential threat and carrying out task-relevant cognitive goals (i.e. directing attention away from threat) (Etkin, Egner, Peraza, Kandel, \& Hirsch, 2006).

Notably, anxious youth (Beesdo et al., 2009; Guyer et al., 2008) and adults (Etkin \& Wager, 2007) exhibit increased amygdala reactivity when performing tasks with threatening stimuli and less 
activation in the ACC while performing tasks that require participants to direct their attention away from emotional stimuli (Bishop, Duncan, Brett, \& Lawrence, 2004; Klumpp et al., 2011). Moreover, in a task developed in our laboratory, we had previously shown that when participants are instructed to match emotional faces (explicit emotion processing) or match shapes in the context of emotional face distractors (implicit emotion processing), both youth (Swartz et al., 2014) and adults (Klumpp, Post, Angstadt, Fitzgerald, \& Phan, 2013) with anxiety disorders, compared with healthy controls, exhibit less ACC activation during implicit versus explicit matching.

While these previous studies have generally been consistent in supporting this neuropathophysiological model in anxiety disorders (i.e. enhanced amygdala activation during explicit emotion processing and decreased prefrontal activation when required to direct attention away from emotional stimuli), surprisingly few studies have examined how neural correlates of these processes relate to anxiety treatment outcome. In one study of adults with social anxiety disorder, greater activation of the dorsal ACC during implicit emotion processing (i.e. matching shapes in the context of emotional face distractors) predicted better response to CBT (Klumpp, Fitzgerald, Angstadt, Post, \& Phan, 2014). However, only two previous fMRI studies have examined predictors of treatment response in youth with anxiety disorders, and both of these studies focused solely on explicit emotion processing. In one study, greater amygdala activation in response to viewing fearful faces predicted better treatment response among a sample of anxious youth (McClure et al., 2007). More recently, using a different task that probed explicit emotional processing only, we found that greater activation in dorsolateral and ventrolateral $\mathrm{PFC}$ while processing threatening faces predicted greater response to CBT and SSRI treatment (Kujawa, Swain, et al., 2016).

The primary aim of this study was to extend these previous studies by examining neural correlates of both explicit and implicit emotion processing in relation to treatment outcome for pediatric anxiety. This two-site study was modeled after the Child/ Adolescent Anxiety Multimodal Study (Compton et al., 2010) in that it included children and adolescents across a large span of development (7-19 years) with primary diagnoses of generalized anxiety disorder (GAD) and/or social anxiety disorder (SAD), two of the most common anxiety disorders in youth (Mohatt et al., 2014), treated with either sertraline (i.e. SSRI) or psychotherapy (i.e. CBT). Prior to treatment, participants completed the Emotional Faces Shifting Attention Task (EFSAT; Klumpp, Angstadt, \& Phan, 2012; Klumpp et al., 2013, 2014; Swartz et al., 2014) in which images comprising a trio of geometric shapes (circles, rectangles, triangles) were presented alongside a trio of emotional facial expressions (angry, fearful, happy), and the participant was prompted to match either faces or shapes. The task provides a measure of neural correlates of both explicit and implicit emotional processing, as the instruction to 'Match Shapes' requires participants to direct attention away from emotional faces, whereas 'Match Faces' requires individuals to direct attention toward emotional faces.

Based on the findings that patients with anxiety disorders exhibit less ACC recruitment in the presence of angry, fearful, or happy face distractors (i.e. Match Shapes > Match Faces; Klumpp et al., 2013; Swartz et al., 2014), we expected that recruitment of the ACC during implicit emotion processing (Match Shapes) would influence response to treatment. Regarding the direction of this effect, although we previously demonstrated that greater dorsal ACC activation during implicit processing predicts better response to CBT among anxious adults (Klumpp et al., 2014), studies examining attentional biases in children at the behavioral level suggest the opposite pattern. For example, children who experience difficulty diverting their attention away from threatrelevant stimuli, perform better with CBT (Waters, Mogg, \& Bradley, 2012). This finding is consistent with studies suggesting that attentional biases improve with anxiety treatment (Pishyar, Harris, \& Menzies, 2008) and that the therapeutic effects of anxiety treatment are mediated by early changes in attentional bias (Abend \& Bar-Haim, 2013). Because of these mixed findings, no specific hypotheses were generated regarding the direction of the ACC response during implicit emotion processing and its relation to treatment response in youth with anxiety. Regarding explicit emotion processing (Match Faces), consistent with the two previous pediatric anxiety treatment outcome studies (cf. Kujawa, Swain, et al., 2016; McClure et al., 2007), we predicted that greater activation of the dorsolateral and ventrolateral PFC and the amygdala would be related to better anxiety treatment response.

\section{Methods \\ Participants}

Participants were part of a pediatric anxiety treatment study at the University of Michigan (UM) and the University of Illinois at Chicago (UIC). Participants in the study were youth between the ages of 7 and 19 with primary diagnoses of GAD or social anxiety disorder. The Schedule of Affective Disorders and Schizophrenia for School-Age Children (Kaufman et al., 1997) diagnostic interview was used to assess for diagnoses by master's- or doctoral-level clinicians (see Kujawa, MacNamara, Fitzgerald, Monk, \& Phan, 2015 for more details). Exclusion criteria included history of bipolar disorder, schizophrenia, intellectual disability, pervasive development disorders, current substance use disorders, severe depression, or suicidal ideation. Participants with secondary comorbid anxiety, depressive, or externalizing disorders were included in the study (see Participant Characteristics). Participants were not 
taking psychotropic medications or in psychotherapy for at least 4 weeks prior to the initial assessment.

A total of 44 anxious youth completed the fMRI Emotional Faces Shifting Attention Task (EFSAT), followed by a minimum of 10 weeks of pharmacotherapy or psychotherapy. Data from six participants were excluded for movement during the fMR ( $>3 \mathrm{~mm}$ in any one direction across each functional run) and one participant was excluded for low accuracy on the task $(<20 \%)$, leaving a total sample of 37 youth with pretreatment fMRI and pre- and posttreatment clinical measures $(n=21$ who received SSRI treatment; $n=16$ who received CBT treatment). The included sample was $59.5 \%$ female; $73.0 \%$ Caucasian, 8.1\% African American, 5.4\% Asian, and 13.5\% multiracial; $10.8 \%$ identified as Hispanic/Latino.

\section{Procedure}

Procedures were approved by the institutional review boards at both UIC and UM. Participants completed the clinical interview and pretreatment symptom measures during the initial visit. Participants then completed the fMRI scan (within 1 week of initial interview). At UM, participants were offered and self-selected treatment with SSRI or CBT. At UIC, participants were initially randomly assigned to receive either an SSRI or CBT, but could opt to switch from SSRI to CBT due to intolerable side effects. SSRI treatment consisted of 12 weeks of sertraline prescribed by a child psychiatrist during medication management sessions, beginning with a dose of 12.5 or $25 \mathrm{mg} /$ day and in a flexible-dosing design increasing on subsequent visits up to $200 \mathrm{mg} /$ day based on tolerability and treatment response. CBT was delivered through weekly 60-minute sessions (up to a maximum of 18 sessions) by a master's- or doctoral-level therapist. Treatment followed an established manualized CBT intervention (i.e. Coping Cat, C.A.T Project) for pediatric anxiety (Kendall, Choudhury, Hudson, \& Webb, 2002; Kendall \& Hedtke, 2006).

\section{Measures}

Emotional faces shifting attention task (EFSAT; Klumpp et al., 2012, 2013, 2014; Swartz et al., 2014). A trial of the EFSAT consists of three faces in a triangular configuration, and three shapes in an upside-down

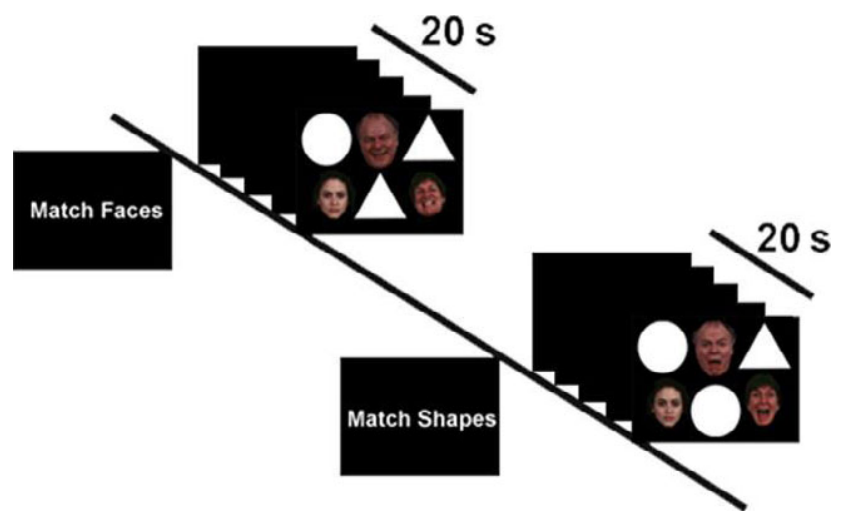

Figure 1 Schematic of exemplar 'Match Faces' and 'Match Shapes' blocks in the Emotional Faces Shifting Attention Task (EFSAT). Trials were presented in block format, and participants were instructed at the beginning of each block to either match faces or match shapes for that block. The match faces condition requires attending to the emotional faces, whereas the match shapes condition requires performing the shape-matching task in the context of emotional face distractors [Colour figure can be viewed at wileyonlinelibrary.com] triangular configuration (Figure 1). During the faces condition, participants were instructed to identify which face on the bottom row matched the emotion of the target face on the top. During the shapes condition, participants were instructed to match shapes on the top row with the target shape on the bottom. Participants completed two runs, for a total of 18 faces blocks and 18 shapes blocks with six blocks of each condition: match angry faces, fear faces, and happy faces, and match shapes with angry faces, fear faces, and happy faces as distractors. Each block began with a 4-s cue to either match faces or match shapes followed by the four sequential matching trials, each lasting $4 \mathrm{~s}$.

fMRI data acquisition and processing. MRI data were collected on 3 Tesla GE scanners with 8-channel head coils at both sites. At UM, functional data were collected with a gradient-echo reverse spiral acquisition with the following parameters: repetition time $(\mathrm{TR})=2 \mathrm{~s}$, echo time $(\mathrm{TE})=30 \mathrm{~ms}$, flip angle $=90^{\circ}$, field of view (FOV) $=22 \times 22 \mathrm{~cm}$, acquisition matrix $64 \times 64,3-\mathrm{mm}$ slice thickness, and 43 axial slices. At UIC, functional data were acquired using gradient-echo echoplanar imaging (EPI) sequence with the following parameters: $\mathrm{TR}=2 \mathrm{~s}, \quad \mathrm{TE}=\operatorname{minFull} \quad[\sim 25 \mathrm{~ms}], \quad$ flip $\quad$ angle $=90^{\circ}$, FOV $=22 \times 22 \mathrm{~cm}$, acquisition matrix $64 \times 64,3-\mathrm{mm}$ slice thickness, and 44 axial slices.

Functional images were preprocessed in SPM8 (Wellcome Trust Centre for Neuroimaging, http://www.fil.ion.ucl.ac.uk/ $\mathrm{spm} /$ ) for slice timing correction, image normalization, and resampling at a $2 \times 2 \times 2 \mathrm{~mm}^{3}$ voxel size and 8 - $\mathrm{mm}$ Gaussian smoothing kernel. Condition effects were modeled at the individual subject level using the general linear model, and the nuisance regressors for six motion parameters were included to correct for motion artifacts. For each participant, contrast images of brain activity were generated for secondlevel analysis.

Response to treatment. To assess severity of anxiety symptoms at pre- and posttreatment, participants were administered the Pediatric Anxiety Rating Scale (PARS; Research Units, 2002). The PARS was completed at the initial screening visit and at the final treatment session. Greater PARS change scores (i.e. pretreatment minus posttreatment) indicate greater response to treatment.

\section{Data analysis}

Consistent with previous studies in our laboratory using the EFSAT task to predict treatment response (Klumpp et al., 2014), a one-sample $t$-test with change in PARS scores as a covariate of interest was performed in SPM8 for the March Face $>$ Match Shape contrast. To control for methodological and demographic variables, site/scanner (UM or UIC), treatment (SSRI or CBT), and age were included as covariates in the second-level analysis. Based on a priori regions of interest from our hypotheses, we focused on the bilateral amygdala and the dorsolateral and ventrolateral PFC using the Wake Forest University Pickatlas (WFU Pickatlas) (Maldjian, Laurienti, Kraft, \& Burdette, 2003). In order to obtain an ROI for the rostral portion of the ACC, the Anterior Cingulate Region was intersected with the medial frontal region (Swartz et al., 2014) defined by the Automated Anatomical Labeling (AAL) atlas. Activations were deemed as significant using small-volume correction (SVC) and family-wise error (FWE) corrected $p<.05$. For completeness, we also examine activation clusters outside a priori ROIs at the whole-brain level using FWE correction at $p<.05$.

Exploratory analyses were conducted to investigate emotion-specific effects. Specifically, activation (beta weights) from a 10-mm diameter sphere around peak voxels surviving smallvolume correction and FWE (ROI regions) for the Match 
Face $>$ Match Shape contrast were extracted using MarsBar (Brett, Anton, Valabregue, \& Poline, 2002) and exported into SPSS for the separate emotion contrasts: Match Shape $_{[\text {Angry] }}>$ Match Face $_{[\text {Angry] }}$, Match Shape $_{[\text {Fear] }}>$ Match Face $_{[\text {Fear] }}$, and Match Shape ${ }_{[\text {Happy] }}>$ Match Face $_{[\text {Happy] }}$. In SPSS, a generalized linear model was then conducted with activation for each emotion type (angry, fear, and happy) serving as the within-subject variables and PARS pre-to-post change, site/scanner (UM or UIC), treatment (SSRI or CBT), and age as continuous between-subjects variables. Greenhouse-Geisser corrections were used in cases in which the sphericity assumption was violated and Bonferroni procedures were used to correct for multiple comparisons.

\section{Results \\ Participant characteristics}

With regard to clinical disorders, $67.6 \%$ of the sample had current diagnoses of GAD, 59.5\% SAD, and $10.8 \%$ separation anxiety disorder. In addition, $10.8 \%$ had comorbid separation anxiety disorder, $10.8 \%$ panic disorder, $2.7 \%$ obsessive-compulsive disorder, $18.9 \%$ specific phobia, 5.4\% depression, and $16.2 \%$ ADHD. Anxiety severity (i.e. PARS) decreased following treatment, $t(36)=14.07, p<$ .001 , mean difference $=13.54$. With regard to treatment response, $79.1 \%$ of the sample responded to treatment and $69.8 \%$ showed evidence of remission, as indicated by at least a $35 \%$ and $50 \%$ decrease in PARS, respectively (Caporino et al., 2013).

Participant characteristics by treatment group are presented in Table 1. CBT and SSRI groups did not significantly differ on PARS, age, or distribution of sex, race, IQ, or primary diagnosis ( $p$ s > .13); however, the CBT group completed more sessions than the SSRI group, $t(35)=-5.15, p<.001$. Study sites did not significantly differ on treatment modality (UM: $n=10$ CBT, $n=11$ SSRI; UIC: $n=6$ CBT, $n=10$ SSRI), PARS posttreatment or change, age, number of sessions, or primary diagnoses ( $p s>.09$ ). Compared with the sample recruited at UM, the UIC sample had greater pretreatment PARS scores, $t(35)=-2.72, \quad p=.01$, and included a greater proportion of males, $\chi^{2}(1)=5.64, p=.02$, and participants who identified as Hispanic/Latino, $\chi^{2}(1)=5.89, p=.05$.

\section{Behavioral performance}

Behavioral data were lost for one participant due to a technical error. To examine the influence of emotion (angry, fear, and happy) and condition (shapes vs. faces) on accuracy and $\mathrm{RT}$, a repeated-measures ANOVA was conducted. Results revealed a main effect of condition, $F(1,34)=13.64, p<.001$, with accuracy being higher for matching shapes $(M=84.92, \quad S E=2.10) \quad$ compared with matching faces $(M=79.22, \quad S E=2.31)$ across participants. There was also a main effect of condition for RT, $F(1,34)=32.83, p<.001$, with slower responses when matching faces $(M=1625.06, \quad S E=98.17)$
Table 1 Participant characteristics by treatment group [selective serotonin reuptake inhibitor (SSRI) or cognitive behavioral therapy (CBT)] and study site [University of Michigan (UM) or University of Illinois at Chicago (UIC)]

\begin{tabular}{|c|c|c|c|c|c|}
\hline & \multicolumn{2}{|c|}{ SSRI $(n=21)$} & \multicolumn{3}{|c|}{$\operatorname{CBT}(n=16)$} \\
\hline & Mean & $S D$ & \multicolumn{2}{|c|}{ Mean } & $S D$ \\
\hline Age & 14.43 & 2.87 & \multicolumn{2}{|c|}{14.50} & 3. \\
\hline Number of sessions & 11.90 & 0.44 & \multicolumn{2}{|c|}{14.87} & 2.6 \\
\hline Pretreatment PARS & 23.62 & 5.06 & \multicolumn{2}{|c|}{22.13} & 3. \\
\hline Posttreatment PARS & 9.57 & 5.72 & \multicolumn{2}{|c|}{9.25} & 6. \\
\hline PARS change & 14.05 & 6.31 & \multicolumn{2}{|c|}{12.88} & 5. \\
\hline \multirow[t]{2}{*}{ WASI IQ estimate } & 110.41 & 11.24 & \multicolumn{2}{|c|}{109.38} & 11.9 \\
\hline & & $N$ & $\%$ & $N$ & $\%$ \\
\hline Female & & 11 & 52.4 & 11 & 68 \\
\hline Primary GAD & & 12 & 57.1 & 9 & 56 \\
\hline Primary social anxiet & lisorder & 9 & 42.9 & 7 & 43 \\
\hline
\end{tabular}

\begin{tabular}{|c|c|c|c|c|c|}
\hline & \multicolumn{2}{|c|}{$\mathrm{UM}(n=21)$} & \multicolumn{3}{|c|}{$\mathrm{UIC}(n=16)$} \\
\hline & Mean & $S D$ & \multicolumn{2}{|c|}{ Mean } & $S D$ \\
\hline Age & 14.09 & 2.79 & \multicolumn{2}{|c|}{14.94} & 3.07 \\
\hline Number of sessions & 12.81 & 1.94 & \multicolumn{2}{|c|}{13.69} & 2.63 \\
\hline Pretreatment PARS & 21.38 & 4.14 & \multicolumn{2}{|c|}{25.06} & 4.01 \\
\hline Posttreatment PARS & 7.90 & 5.61 & \multicolumn{2}{|c|}{11.44} & 5.69 \\
\hline PARS change & 13.48 & 6.35 & \multicolumn{2}{|c|}{13.63} & 5.33 \\
\hline \multirow[t]{2}{*}{ WASI IQ estimate } & 110.76 & 11.34 & \multicolumn{2}{|c|}{109.00} & 11.83 \\
\hline & & $N$ & $\%$ & $N$ & $\%$ \\
\hline \multicolumn{2}{|l|}{ Gender (Female) } & 16 & 76.2 & 6 & 37.5 \\
\hline \multicolumn{2}{|c|}{ Primary GAD } & 13 & 61.9 & 8 & 50.0 \\
\hline \multicolumn{2}{|c|}{ Primary social anxiety disorder } & 8 & 38.1 & 8 & 50.0 \\
\hline
\end{tabular}

compared with matching shapes $(M=1269.11$, $S E=92.15)$. None of the other main effects or interactions were significant for accuracy or RT (lowest $p=.09$ ).

\section{fMRI predictors of treatment response}

Results of the ROI one-sample $t$-test revealed that lower activation of superior frontal gyrus $[(12,54,20)$, $t$-value $=3.47, \quad$ volume $=2,896 \mathrm{~mm}^{3}, \quad$ svccorrected $p=.03$ ] - a large cluster encompassing the dorsal ACC and ventromedial PFC - during the Match Shape $>$ Match Face contrast predicted a greater reduction in anxiety symptoms pre-to-post treatment. No other regions outside the SFG were associated with change in symptoms for the Match Shape $>$ Match Face contrast within ROIs or for the whole-brain analysis.

Exploratory analyses were then conducted to examine emotion-specific effects. We extracted beta weights from a spherical ROI around the peak voxel for the SFG cluster (12 54 20) for the Match Shape $_{[\text {Angry] }}>$ Match Face $_{[\text {Angry] }}$, Match Shape [Fear] $>$ Match Face $_{[\mathrm{Fear}]}$, and Match Shape HHappy] $>$ Match Face $_{\text {[Happy] }}$ contrasts. A generalized linear model was then conducted with SFG activation for each emotion 
type (angry, fear, and happy) serving as the withinsubject variables and PARS pre-to-post change, site/ scanner (UM or UIC), treatment (SSRI or CBT), and age as continuous between-subjects variables. Results revealed a significant Emotion $\times$ PARS preto-post Change interaction for SFG activation, $F(2$, $31)=3.35, p=.04, n p^{2}=.10$. To follow-up this interaction, partial correlations between SFG activation for each emotion type and PARS pre-to-post change were calculated controlling for treatment modality, site, and age. Results revealed a significant negative correlation between PARS pre-to-post change and SFG activation for the Match Shape $_{[\text {Fear] }}>$ Match Face $_{[\text {Fear }]}$ contrast $(r=-.47$, $p<.01$; Figure 2). No significant relations with PARS pre-to-post change were observed for the Match Shape [Angry] $>$ Match Face $_{\text {[Angry] }}$ or Match Shape $_{[\text {Happy] }}>$ Match Face $_{[\text {Happy] }}$ contrasts (lowest $p=.12)$. The correlation between PARS pre-to-post change and SFG activation for the Match Shape $_{[\text {Fear }]}>$ Match Face $_{[\text {Fear }]}$ contrast remained significant after adjusting for youth's initial anxiety symptom severity, $\left(\right.$ PARS $\left._{\text {PreTx }}\right), r=-.39, p=.02$ and IQ (WASI), $r=-.41, p=.02$. None of the interactions with participant's age and gender, study site, and treatment were significant (lowest $p=.09$ ). We also examined whether participant's primary anxiety diagnosis moderated the relation between SFG activation during implicit fear processing and treatment response; none of these analyses were significant (lowest $p=.21$ ).
Finally, because the shapes $>$ faces contrast can be interpreted as decreased activation when matching shapes (implicit emotion processing) or increased activation when matching faces (explicit emotion processing), we extracted beta weights from a spherical ROI around the peak voxel for the SFG for Match Shape $_{[\text {Fear }]}>$ Baseline (activation across all conditions) and Match Face $_{[\mathrm{Fear}]}>$ Baseline contrasts. Partial correlations in SPSS between brain activation and PARS pre-to-post change were then calculated controlling for age, treatment modality, and site. As shown in Figure 2, SFG activation during implicit fear processing was negatively correlated with PARS pre-to-post change $(r=-.38, p=.02)$; however, the Match Face $_{[\mathrm{Fear}]}>$ Baseline contrast was not related to PARS change $(r=.12, p=.48)$.

\section{Discussion}

This study used fMRI to examine potential brain predictors of treatment response for pediatric anxiety. Given the well-established link between attentional biases and anxiety disorders, (e.g. Bar-Haim et al., 2007; Bishop, 2007; Pine, 2007), this study examined the neural correlates of directing attention toward (explicit emotion processing) and away (implicit emotion processing) from emotional stimuli in relation to pediatric anxiety treatment response. Results revealed that reduced activation in SFG, encompassing the dorsal ACC and dorsomedial PFC, during implicit emotion processing predicted a

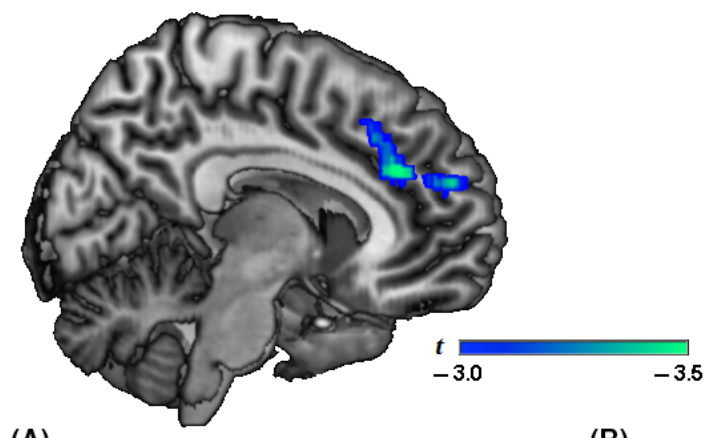

(A)

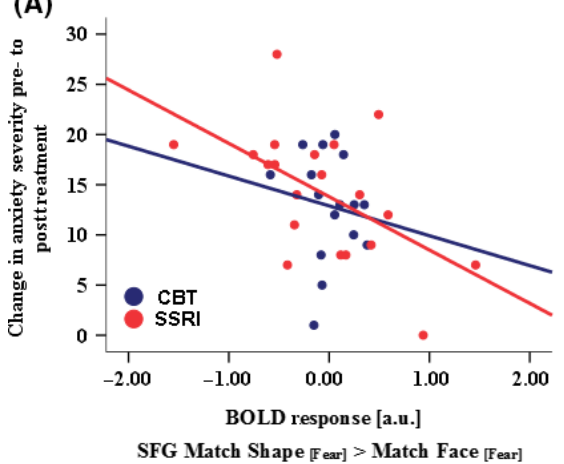

(B)

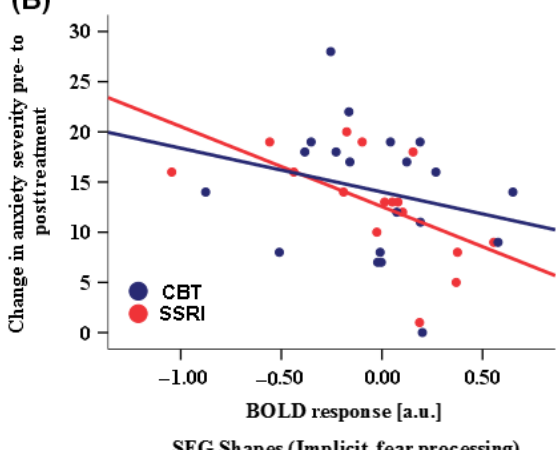

SFG Shapes (Implicit fear processing)

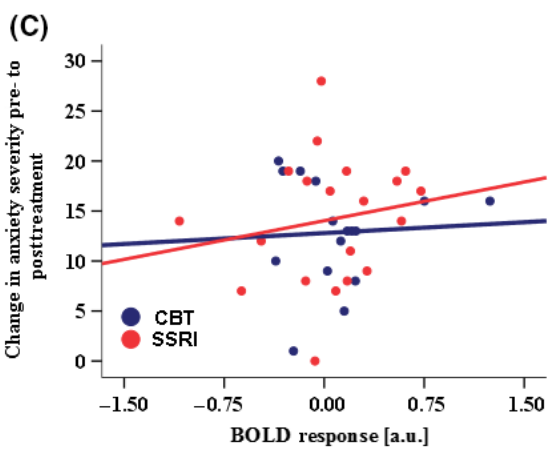

SFG faces (Explicit fear processing)

Figure 2 Exploratory analyses findings showing that reduced superior frontal gyrus, including the dorsal anterior cingulate cortex and dorsomedial prefrontal cortex, activation during processing of shapes in the context of fearful face distractors predicted greater change in anxiety severity, measured via the Pediatric Anxiety Rating Scale, pretreatment to posttreatment. Association between anxiety symptom change, measured via the Pediatric Anxiety Rating Scale, pre-to-post treatment and superior frontal gyrus (SFG) activation (mean blood-oxygen-level-dependent parameter estimates; a.u. = arbitrary units) during (A) Match Shapes [Fear] $>$ Match Faces [Fear], (B) Match Shape ${ }_{[\mathrm{Fear}]}>$ Baseline (Implicit Processing), and (C) Match Faces ${ }_{[\mathrm{Fear}]}>$ Baseline (Explicit Processing) 
greater reduction in anxiety severity pre- to posttreatment. Exploratory analyses revealed that this effect appeared to be driven by implicit fear processing and was not observed for matching faces in the context of happy or angry face distractors. We found no evidence for these effects being moderated by type of treatment or primary diagnosis, suggesting similar effects across SSRI and CBT interventions, as well as diagnoses of GAD and social anxiety disorder.

The ACC plays a crucial role in attention-emotion processes (Bush, Luu, \& Posner, 2000; Carter et al., 1998), and the dorsal ACC, in particular, has been linked to conflict monitoring and cognitive appraisal processes (Etkin, Egner, \& Kalisch, 2011). In children and adults with anxiety, the ACC has been linked to ability to direct attention away from stimuli when threatening material is presented (e.g. Bishop et al., 2004; Klumpp et al., 2011, 2012; Swartz et al., 2014). Thus, children who recruit less dorsal ACC during attempts to process shapes when emotional stimuli are presented may have more to gain from CBT and SSRI treatment due to preexisting deficits in attentional control and implicit regulatory ability. This interpretation is consistent with recent evidence that the therapeutic effects of anxiety treatment are mediated by early changes in attentional bias (Abend \& Bar-Haim, 2013) and from behavioral studies suggesting that attentional control improves with anxiety treatment (Pishyar et al., 2008). In addition, the current findings parallel recent research using a dot-probe task with children suggesting that anxious youth who exhibit pretreatment attention biases toward threatening stimuli show greater reductions not only in anxiety symptom severity but also in the likelihood of meeting diagnostic criteria for anxiety disorders following treatment (Waters et al., 2012). These findings provide preliminary evidence at the neural level that youth who recruit less ACC during attempts to direct their attention away from emotional stimuli may benefit the most from anxiety treatment, potentially due to having more to gain in treatment. Future studies are needed to determine whether activation of ACC during the processing of neutral information in the context of emotional distractors improves pre-topost treatment among youth with anxiety disorders.

Notably, we previously demonstrated in adults that greater dorsal ACC during implicit emotion processing was related to greater anxiety symptom reduction following CBT (Klumpp et al., 2014). One possible explanation for the discrepant findings is that the current sample focused on response to both CBT and SSRI treatment, whereas the previous adult study focused solely on response to CBT. Importantly, previous studies suggest that distinct patterns of ACC activation (i.e. hypo vs. hyper) may predict response to CBT versus medication in depression (Ball, Stein, \& Paulus, 2014); thus, it is possible that current findings are primarily driven by those treated with SSRI and our analyses may be underpowered to detect differences between the two treatments. Alternatively, there may be differences in the direction of ACC activation depending on age and development. For example, recent work suggests that anxiety is characterized by altered developmental patterns of amygdala connectivity with ACC from childhood into young adulthood (Kujawa, Wu, et al., 2016). In addition, a separate study shows that age moderates the link between anxiety and the errorrelated negativity, an event-related potential component that is generated in the ACC (Meyer, Weinberg, Klein, \& Hajcak, 2012). Among older children, a larger ERN is significantly related to increased anxiety; however, the relationship between ERN and anxiety appears to be opposite among younger children. Thus, it will be important for future, larger studies to examine how developmental differences impact the relation between ACC activation during emotion processing and treatment response.

Decreased dorsomedial PFC activation during implicit emotion processing also predicted a significant reduction in anxiety symptoms pre-to-post treatment. The dorsomedial PFC is part of an extended medial prefrontal network involved in assessing the value and valence of stimuli and generating adaptive responses that guide behavior (Etkin et al., 2011; Price \& Drevets, 2010). Thus, decreased recruitment of this specific region in response to disengaging from emotional information suggests that some individuals may have been more likely to improve if they had a reduced capacity to regulate their responses when attempting to direct their attention away from the emotional stimuli prior to beginning treatment.

This study extends previous work on pediatric anxiety treatment response by examining the influence of implicit emotion processing. The two previous studies utilizing fMRI to examine predictors of anxiety treatment outcome focused solely on explicit emotion processing and found that greater amygdala (McClure et al., 2007) and dorsolateral and ventrolateral PFC (Kujawa, Swain, et al., 2016) activation in response to viewing threatening faces predicted better treatment response among anxious youth. In this study, exploratory post hoc analyses revealed that treatment response was significantly related to ACC activation during implicit, but not explicit, fear processing. However, the baseline contrast used in these analyses reflects average activation across the entire task, which involves explicit emotion processing. Therefore, future research would benefit from including a separate neutral condition (e.g. 'match houses') in the EFSAT task to more definitively test whether ACC activation is specific to explicit versus implicit processing in relation to pediatric anxiety treatment response.

Results from this study also showed that the majority of the current sample $(79.1 \%)$ showed a $35 \%$ or greater reduction in PARS severity, which 
has been associated with treatment response (Caporino et al., 2013). This is noteworthy as this response rate is higher than rates reported in large randomized controlled trials of CBT and SSRI in anxious youth (e.g. Walkup et al., 2008). This is likely due to the current study only including youth who completed treatment, the fMRI scan, and had pre- and posttreatment clinical measures. Future research is needed to evaluate whether neural measures may be useful in predicting treatment engagement and tolerability in children and adolescents with anxiety disorders.

The current findings should be interpreted in the context of several limitations. First, the small sample size may have limited our ability to detect moderators (i.e. age, sex, and treatment type) of treatment response. Second, the study sample is heterogeneous with regard to primary diagnoses; although primary diagnoses of SAD and GAD did not moderate the effects, future studies with larger sample sizes are needed to determine if differences are specific to certain anxiety diagnostic groups. Third, the exploratory analytical approach used in this study to examine emotion-specific effects was circular (i.e. nonindependent; for a review, see Kriegeskorte, Lindquist, Nichols, Poldrack, \& Vul, 2010), as the extracted beta weights for the SFG cluster were derived from the Match Shape $>$ Match Face contrast. Therefore, due to potential noise bias, we cannot definitively conclude that the current findings were driven by fearful face distractors. Finally, the study was completed across two sites. Although our findings did not reveal a significant effect of site as a moderator, nonspecific factors from each site, such as patient preference versus random assignment, may have contributed to the findings.

\section{Conclusion}

This is the first study to examine the neural correlates of implicit emotion processing in relation to pediatric anxiety treatment response. The findings suggest that less recruitment of SFG, including the dorsal ACC and dorsomedial PFC, during implicit emotion processing predicts a greater reduction in anxiety symptoms pre-to-post treatment. Youth who exhibit reduced activation in these areas while matching shapes in the context of emotional face distractors may have more to gain from CBT and SSRI treatment due to preexisting deficits in attentional control. These findings suggest that neuroimaging may be a useful tool for predicting which youth are most likely to benefit from anxiety treatment.

\section{Acknowledgements}

This study was supported by the National Institute of Mental Health Grant R01-MH086517 to C.S.M. and K.L.P. K.L.B. and A.K. are supported by the National Institute of Mental Health Grant T32- MH067631 (Training in the Neuroscience of Mental Health; PI: Mark Rasenick). The authors declare that they have no competing or potential conflicts of interest to declare. They thank James Swain, Gregory Hanna, Elizabeth Koschmann, David Simpson, and Sucheta Connolly for their help in providing therapeutic services for this study.

\section{Correspondence}

Katie L. Burkhouse, Department of Psychiatry, University of Illinois at Chicago, $1747 \mathrm{~W}$ Roosevelt Road, Chicago, IL 60608, USA; Email: kburkhouse@psych. uic.edu

\section{Key points}

- Approximately $40 \%-45 \%$ of youth with anxiety disorders do not achieve remission following treatment, highlighting the need to identify predictors of treatment response.

- This study examined the neural correlates of directing attention toward and away from emotional faces in relation to pediatric anxiety treatment response.

- Less recruitment of superior frontal gyrus, including the dorsal anterior cingulate cortex, during implicit emotion processing predicted a greater reduction in anxiety symptoms pre-to-post treatment.

- Youth who exhibit reduced activation in these areas while matching shapes in the context of emotional face distractors may have more to gain from CBT and SSRI treatment due to preexisting deficits in attentional control.

- Neuroimaging may be a useful tool for predicting which youth are most likely to benefit from anxiety treatment.

\section{References}

Abend, R., \& Bar-Haim, Y. (2013). Threat-related attention bias in the early stages of cognitive-behavior therapy action for panic disorder. Biological Psychiatry, 73, 1041-1042.
Ball, T.M., Stein, M.B., \& Paulus, M.P. (2014). Toward the application of functional neuroimaging to individualized treatment for anxiety and depression. Depression and Anxiety, 31, 920-933. 
Bar-Haim, Y., Lamy, D., Pergamin, L., Bakermans-Kranenburg, M.J., \& Van Ijzendoorn, M.H. (2007). Threat-related attentional bias in anxious and nonanxious individuals: A meta-analytic study. Psychological Bulletin, 133, 1-24.

Beesdo, K., Lau, J.Y., Guyer, A.E., McClure-Tone, E.B., Monk, C.S., Nelson, E.E., ... \& Ernst, M. (2009). Common and distinct amygdala-function perturbations in depressed vs anxious adolescents. Archives of General Psychiatry, 66, 275-285.

Bishop, S.J. (2007). Neurocognitive mechanisms of anxiety: An integrative account. Trends in Cognitive Sciences, 11, 307316.

Bishop, S., Duncan, J., Brett, M., \& Lawrence, A.D. (2004). Prefrontal cortical function and anxiety: Controlling attention to threat-related stimuli. Nature Neuroscience, 7, 184188.

Brett, M., Anton, J.L., Valabregue, R., \& Poline, J.B. (2002). Region of interest analysis using an SPM toolbox. Presented at the 8th International Conference on Functional Mapping of the Human Brain; 2-6 June 2002; Sendai, Japan. Available on CD-ROM in NeuroImage, Vol 16, No 2.

Bush, G., Luu, P., \& Posner, M.I. (2000). Cognitive and emotional influences in anterior cingulate cortex. Trends in Cognitive Sciences, 4, 215-222.

Caporino, N.E., Brodman, D.M., Kendall, P.C., Albano, A.M., Sherrill, J., Piacentini, J., .. \& \& Rynn, M. (2013). Defining treatment response and remission in child anxiety: Signal detection analysis using the pediatric anxiety rating scale. Journal of the American Academy of Child and Adolescent Psychiatry, 52, 57-67.

Carter, C.S., Braver, T.S., Barch, D.M., Botvinick, M.M., Noll, D., \& Cohen, J.D. (1998). Anterior cingulate cortex, error detection, and the online monitoring of performance. Science, 280, 747-749.

Clark, D.A., Beck, A.T., \& Alford, B.A. (1999). Scientific foundations of cognitive theory and therapy of depression. Hoboken, NJ: John Wiley \& Sons Inc.

Compton, S.N., Walkup, J.T., Albano, A.M., Piacentini, J.C., Birmaher, B., Sherrill, J.T., .. . \& Iyengar, S. (2010). Child/ adolescent anxiety multimodal study (CAMS): Rationale, design, and methods. Child and Adolescent Psychiatry and Mental Health, 4, 1-15.

Costello, E.J., Mustillo, S., Erkanli, A., Keeler, G., \& Angold, A. (2003). Prevalence and development of psychiatric disorders in childhood and adolescence. Archives of General Psychiatry, 60, 837-844.

Etkin, A., Egner, T., \& Kalisch, R. (2011). Emotional processing in anterior cingulate and medial prefrontal cortex. Trends in Cognitive Sciences, 15, 85-93.

Etkin, A., Egner, T., Peraza, D.M., Kandel, E.R., \& Hirsch, J. (2006). Resolving emotional conflict: A role for the rostral anterior cingulate cortex in modulating activity in the amygdala. Neuron, 51, 871-882.

Etkin, A., \& Wager, T.D. (2007). Functional neuroimaging of anxiety: A meta-analysis of emotional processing in PTSD, social anxiety disorder, and specific phobia. American Journal of Psychiatry, 164, 1476-1488.

Guyer, A.E., Lau, J.Y., McClure-Tone, E.B., Parrish, J., Shiffrin, N.D., Reynolds, R.C., .. \& \& Ernst, M. (2008). Amygdala and ventrolateral prefrontal cortex function during anticipated peer evaluation in pediatric social anxiety. Archives of General Psychiatry, 65, 1303-1312.

Kaufman, J., Birmaher, B., Brent, D., Rao, U.M.A., Flynn, C., Moreci, P., ... \& Ryan, N. (1997). Schedule for affective disorders and schizophrenia for school-age children-present and lifetime version (K-SADS-PL): Initial reliability and validity data. Journal of the American Academy of Child and Adolescent Psychiatry, 36, 980-988.

Kendall, P.C., Choudhury, S., Hudson, J., \& Webb, A. (2002). The C.A.T. project workbook. Ardmore, PA: Worbook Publishing.
Kendall, P.C., \& Hedtke, K. (2006). Coping cat workbook (2nd edn). Ardmore, PA: Worbook Publishing.

Klumpp, H., Angstadt, M., \& Phan, K.L. (2012). Shifting the focus of attention modulates amygdala and anterior cingulate cortex reactivity to emotional faces. Neuroscience Letters, 514, 210-213.

Klumpp, H., Fitzgerald, D.A., Angstadt, M., Post, D., \& Phan, K.L. (2014). Neural response during attentional control and emotion processing predicts improvement after cognitive behavioral therapy in generalized social anxiety disorder. Psychological Medicine, 44, 3109-3121.

Klumpp, H., Ho, S.S., Taylor, S.F., Phan, K.L., Abelson, J.L., \& Liberzon, I. (2011). Trait anxiety modulates anterior cingulate activation to threat interference. Depression and Anxiety, 28, 194-201.

Klumpp, H., Post, D., Angstadt, M., Fitzgerald, D.A., \& Phan, K.L. (2013). Anterior cingulate cortex and insula response during indirect and direct processing of emotional faces in generalized social anxiety disorder. Biology of Mood and Anxiety Disorders, 3, 1.

Kriegeskorte, N., Lindquist, M.A., Nichols, T.E., Poldrack, R.A., \& Vul, E. (2010). Everything you never wanted to know about circular analysis, but were afraid to ask. Journal of Cerebral Blood Flow and Metabolism, 30, 1551-1557.

Kujawa, A., MacNamara, A., Fitzgerald, K.D., Monk, C.S., \& Phan, K.L. (2015). Enhanced neural reactivity to threatening faces in anxious youth: Evidence from event-related potentials. Journal of Abnormal Child Psychology, 43, 1493-1501.

Kujawa, A., Swain, J.E., Hanna, G.L., Koschmann, E., Simpson, D., Connolly, S., ... \& Phan, K.L. (2016). Prefrontal reactivity to social signals of threat as a predictor of treatment response in anxious youth. Neuropsychopharmacology, 41, 1983-1990.

Kujawa, A., Wu, M., Klumpp, H., Pine, D.S., Swain, J.E., Fitzgerald, K.D., .. \& \& Phan, K.L. (2016). Altered development of amygdala-anterior cingulate cortex connectivity in anxious youth and young adults. Biological Psychiatry: Cognitive Neuroscience and Neuroimaging, 1, 345-352.

Maldjian, J.A., Laurienti, P.J., Kraft, R.A., \& Burdette, J.H. (2003). An automated method for neuroanatomic and cytoarchitectonic atlas-based interrogation of fMRI data sets. NeuroImage, 19, 1233-1239.

McClure, E.B., Adler, A., Monk, C.S., Cameron, J., Smith, S., Nelson, E.E., ... \& Pine, D.S. (2007). fMRI predictors of treatment outcome in pediatric anxiety disorders. Psychopharmacology (Berl), 191, 97-105.

Meyer, A., Weinberg, A., Klein, D.N., \& Hajcak, G. (2012). The development of the error-related negativity (ERN) and its relationship with anxiety: Evidence from 8 to 13 year-olds. Developmental Cognitive Neuroscience, 1, 152-161.

Mohatt, J., Bennett, S.M., \& Walkup, J.T. (2014). Treatment of separation, generalized, and social anxiety disorders in youths. American Journal of Psychiatry, 171, 741-748.

Mychailyszyn, M.P., Méndez, J.L., \& Kendall, P.C. (2010). School functioning in youth with and without anxiety disorders: Comparisons by diagnosis and comorbidity. School Psychology Review, 39, 106-121.

Pine, D.S. (2007). Research review: A neuroscience framework for pediatric anxiety disorders. Journal of Child Psychology and Psychiatry, 48, 631-648.

Pishyar, R., Harris, L.M., \& Menzies, R.G. (2008). Responsiveness of measures of attentional bias to clinical change in social phobia. Cognition and Emotion, 22, 1209-1227.

Price, J.L., \& Drevets, W.C. (2010). Neurocircuitry of mood disorders. Neuropsychopharmacology, 35, 192-216.

Research Units of Pediatric Psychopharmacology Anxiety Study Group (2002). The Pediatric Anxiety Rating Scale (PARS): Development and psychometric properties. Journal of the American Academy of Child and Adolescent Psychiatry, 41, 1061-1069. 
Swartz, J.R., Phan, K.L., Angstadt, M., Klumpp, H., Fitzgerald, K.D., \& Monk, C.S. (2014). Altered activation of the rostral anterior cingulate cortex in the context of emotional face distractors in children and adolescents with anxiety disorders. Depression and Anxiety, 31, 870-879.

Walkup, J.T., Albano, A.M., Piacentini, J., Birmaher, B., Compton, S.N., Sherrill, J.T., ... \& Iyengar, S. (2008). Cognitive behavioral therapy, sertraline, or a combination in childhood anxiety. New England Journal of Medicine, 359, 2753-2766.
Waters, A.M., Mogg, K., \& Bradley, B.P. (2012). Direction of threat attention bias predicts treatment outcome in anxious children receiving cognitive-behavioural therapy. Behaviour Research and Therapy, 50, 428-434.

Accepted for publication: 19 September 2016

First published online: 8 November 2016 